\title{
Artigo/Article
}

\section{Aspectos epidemiológicos e distribuiçãogeográfica da esquistossomose e geo-helmintos, no Estado de Sergipe, de acordo com os dados do Programa de Controle da Esquistossomose}

\author{
Epidemiological characteristics and geographical distribution of schistosomiasis and \\ geohelminths, in the State of Sergipe, according to data from the Schistosomiasis Control \\ Program in Sergipe
}

\begin{abstract}
Carla Virginia Vieira Rollemberg ${ }^{1}$, Cybele Maria Bomfim Santos ${ }^{1}$, Marília Matos Bezerra Lemos Silva ${ }^{2}$, Acacia Maria Barros Souza ${ }^{2}$, Ângela Maria da Silva ${ }^{1}$, José Antônio Pacheco de Almeida ${ }^{2}$, Roque Pacheco de Almeida ${ }^{1,3}$ e Amélia Ribeiro de Jesus ${ }^{1,3}$
\end{abstract}

\begin{abstract}
RESUMO
Introdução: A esquistossomose é endêmica no Brasil, com elevada prevalência no Estado de Sergipe, apesar da existência do Programa de Controle da Esquistossomose (PCE). Métodos: Foi realizado levantamento de dados do PCE-Sergipe de 2005 a 2008. A partir da matriz bruta formulou-se planilha de dados no software Access e analisou-se frequência e distribuição geográfica das infecções por Schistosoma mansoni e outros enteroparasitos. Estes dados foram exportados para o software Spring 5.0.5 para georreferenciamento e confecção de mapas temáticos de distribuição espacial e temporal por ano de avaliação. Resultados: Foram positivos para S. mansoni 13,6\% (14471/106287) de exames nos anos de 2005, 11,2\% (16196/145069) em 2006, 11,8\% (10220/86824) em 2007 e 10,6\% (8329/78859) em 2008. A análise de mapas mostrou elevada prevalência da doença em Sergipe, em particular nos municípios Ilha das Flores, Santa Rosa de Lima, Santa Luzia do Itanhi e São Cristóvão. Além disso, avaliamos a associação entre as frequências dessas doenças parasitárias com indicadores sociais e de desenvolvimento dos diferentes municípios, de acordo com os dados do Instituto Brasileiro de Geografia e Estatística (IBGE) e da Superintendência de Recursos Hídricos (SRH). Observamos que os municípios com prevalência da esquistossomose maior do que $15 \%$ têm menor concentração de rede de esgotos (índice de higiene); $\mathrm{p}=0,05$. Adicionalmente, os municípios com prevalência de infecção por ancilostomídeos maior do que $10 \%$ apresentam um menor IDH educacional; $p=0,04$. Conclusões: Ressalta-se a importância de maior controle dos fatores de risco ambientais e educacionais, na tentativa de reduzir prevalências dessas doenças parasitárias.

Palavras-chaves: Esquistossomose. Schistosoma mansoni. Epidemiologia. Morbidade. Geoprocessamento.
\end{abstract}

\begin{abstract}
Introduction: Schistosomiasis is endemic in Brazil, with high prevalence in the State of Sergipe, despite the existence of the Schistosomiasis Control Program (PCE). Methods: The data from Sergipe's PCE between 2005 and 2008 were surveyed. From the raw information, a database was created on a spreadsheet using the Access software. The frequency and geographic distribution of infections due to Schistosoma mansoni and other intestinal parasites were analyzed. These data were exported to the Spring 5.0.5 software for georeferencing and preparation of thematic maps of the spatial and temporal distribution according to year of evaluation. Results: In 2005, 13.6\% (14,471/106,287) of the tests were positive for S. mansoni, 11.2\% (16,196/145,069) in $2006,11.8 \%(10,220 / 86,824)$ in 2007 and $10.6 \%(8,329 / 78,859)$ in 2008 . Analysis on the maps showed that there was high prevalence of the disease in Sergipe, and particularly in the municipalities of Ilha das Flores, Santa Rosa de Lima, Santa Luzia do Itanhi and São Cristóvão. Furthermore, we evaluated the association between the frequencies of these parasitic diseases and social and developmental indicators in the different municipalities, according to data from the Brazilian Institute for Geography and Statistics (IBGE) and the Department of Water Resources (SRH). We found that municipalities with schistosomiasis prevalence higher than $15 \%$ had lower coverage of sewage systems (hygiene index) $(p=0.05)$. Additionally, municipalities with hookworm prevalence higher than $10 \%$ had lower educational HDI $(p=0.04)$. Conclusions: The importance of greater control over environmental risk and educational factors needs to be emphasized in attempts to reduce the prevalence of these parasitic diseases.
\end{abstract}

Keywords: Schistosomiasis. Schistosoma mansoni. Epidemiology. Morbidity. Geoprocessing.

1. Departamento de Medicina, Universidade Federal de Sergipe, Aracaju, SE. 2. Departamento de Geografia, Universidade Federal de Sergipe, Aracaju, SE. 3. Institutos Nacionais de Ciência e Tecnologia, Instituto de Investigação em Imunologia, Conselho Nacional de Desenvolvimento Científico e Tecnológico, São Paulo, SP.

Endereço para correspondência: Dra Amélia Ribeiro de Jesus. DME/UFS. Rua Claudio Batista s/n, Hospital Universitário, Laboratório de Biologia Molecular, Bairro Sanatório, 49060-100 Aracaju, SE. Tel: 55 79 2105-1806

e-mail: jesus-amelia@uol.com.br

Recebido para publicação em 26/07/2010

Aceito em 06/10/2010

\section{INTRODUÇÃO}

A esquistossomose é uma doença parasitária que afeta em torno de 200 milhões de indivíduos em 76 países da África, Ásia e América. Constitui-se ainda em risco para 650 milhões de pessoas residentes em áreas endêmicas ${ }^{1}$. Em 1975, foi criado no Brasil o Programa Especial de Controle da Esquistossomose (PECE), sendo realizados mais de 12 milhões de tratamentos em todo o país, principalmente na região nordeste. Como resultado desse programa foi possível reduzir o número de portadores, as formas graves da doença e a taxa de mortalidade. No entanto, mesmo com a continuidade do Programa de Controle da Esquistossomose (PCE), em 1996, estimou-se em 7,1 milhões o número de portadores da doença no Brasil ${ }^{2}$, sendo a perspectiva de erradicação da doença ainda um imenso desafio ${ }^{3}$.

O Estado de Sergipe apresenta uma das maiores prevalências do país. Segundo dados do Ministério da Saúde, a média da prevalência de esquistossomose em Sergipe no período de 1980 a 1989 foi de 17,3\%, segunda maior do Brasil, menor apenas do que a do Estado de Alagoas. Considerando-se o período de 1990 a 2002, a média no estado foi de $17,7 \%$, bem acima da média nacional de 9,2\% .

A morbidade da doença, representada principalmente pelas formas mais graves, está associada, entre outros fatores, à intensidade e duração da infecção e à resposta imune do hospedeiro aos antígenos do parasito ${ }^{4-10}$. A carga parasitária, por sua vez, é influenciada pelo grau de contato com a água, reflexo das condições socioeconômicas e culturais da população ${ }^{11,12}$. Deve-se observar ainda que a morbidade da doença pode acarretar prejuízos tanto à cognição e desenvolvimento intelectual das crianças infectadas quanto à produtividade dos trabalhadores ${ }^{13}$.

No Brasil, coinfecções entre Schistosoma mansoni e nematóides intestinais, tais como Ascaris lumbricoides, 
Trichuris trichiura e giardíase, ainda são comuns, especialmente nas zonas rurais pobres. O dimensionamento da prevalência das parasitoses intestinais no Brasil tem sido buscado desde a década de 40. No entanto, essas publicações refletem, em sua maioria, a realidade de pequenas localidades, tornando-se difícil um diagnóstico abrangente. Apesar das frequências pontuais, em diferentes populações, as parasitoses intestinais têm alta prevalência em nosso país. Em 1988, a prevalência no Brasil era de 55,3\% em crianças, sendo que a maior parte era poliparasitada ${ }^{14-16}$.

Apesar de Sergipe ter elevada prevalência da doença e fatores socioeconômicos e ambientais favoráveis à manutenção da infecção por S. mansoni, há uma escassez de dados mais recentes na literatura sobre a situação epidemiológica da doença no estado. Este trabalho tem, assim, por objetivo fazer um levantamento em bases de dados do PCE de todos os casos diagnosticados de infecção por $S$. mansoni e outros enteroparasitos e realizar o mapeamento de casos nos municípios do estado no período de 2005 a 2008, retratando a dinâmica da doença e sua relação com fatores socioeconômicos no estado.

\section{MÉTODOS}

Através de estudo ecológico misto de séries temporais e analítico, este trabalho utiliza a análise espacial para confecção de mapas de distribuição e evolução temporal da esquistossomose no Estado de Sergipe segundo os dados do PCE de 2005 a 2008, e associa a prevalência da esquistossomose e de enteroparasitas a indicadores socioeconômicos ambientais e educacionais segundo dados do Instituto Brasileiro de Geografia e Estatística (IBGE) e da Superintendência de recursos hídricos (SRH).

O PCE coleta dados que são enviados pelos centros de saúde dos municípios que trabalham no controle de endemias. Uma amostra de fezes é coletada de cada paciente e a presença de infecção é baseada no exame microscópico de duas lâminas pelo método de Kato-Katz. O paciente é considerado positivo para S. mansoni, ou outro parasita, se pelo menos um ovo for detectado em quaisquer das lâminas. O médodo Kato-Katz é rápido e de fácil execução, além de possuir como vantagem ser quantitativo, sendo possível calcular o número de ovos por gramas de fezes. O número de ovos encontrados no esfregaço fecal deve ser multiplicado por fator de conversão 23, correspondente ao numero de ovos por gramas de fezes. Segundo a Organização Mundial de Saúde, em 1985, existem três categorias para a intensidade da infecção intestinal (expressas em ovos por gramas de fezes) onde se considera: leve (1-100 ovos por grama de fezes), moderada $(101-400)$ e grave $(>400)^{17}$.

Apesar do método de Kato-Katz ser adequado para a detecção de $S$. mansoni e a maioria dos geo-helmintos, é importante o uso associado de outro método para a redução de falsos negativos nos indivíduos com cargas parasitárias baixas de $S$. mansoni e para identificar outros parasitas como estrongilóides, ancilostomídeos e protozoários.

\section{Coleta de dados e confecção de mapas}

Realizou-se um levantamento de dados do PCE vinculado à Secretaria de Saúde do Estado de Sergipe no período de 2005 a 2008. A partir de matriz bruta de dados no software Access realizouse análises de prevalência e distribuição geográfica das infecções por S. mansoni e geo-helmintos. A análise incluiu o processamento de dados obtidos da base georreferenciada da Superintendência de Recursos Hídricos ${ }^{18}$ aliados às cartas digitais do IBGE, censo de $2003^{15}$. Utilizou-se levantamento e análise dos documentos cartográficos para construção e alimentação de Banco de Dados georreferenciados e confecção de mapas temáticos ${ }^{15}$. Estes dados foram tabulados e exportados para o software Spring 5.1.5 para geração de mapas temáticos, mostrando a distribuição das prevalências de esquistossomose por municípios do estado por ano de avaliação.

Análises de associação entre as infecções por $S$. mansoni e geo-helmintos com marcadores socioeconômicos, ambientais e educacionais

Foram feitas aquisições de índices utilizados como marcadores socioeconômicos e de desenvolvimento social e educacional do Instituto Brasileiro de Geografia e Estatística (IBGE), a exemplo do Índice de Desenvolvimento Humano (IDH), o IDH educacional (IDHE) e a Situação de Higiene. O IDH é um indicador mais completo do que o Produto Interno Bruto (PIB) per capita, o qual considera apenas a dimensão econômica do desenvolvimento, pois este inclui média aritmética simples de três subíndices, referentes às dimensões longevidade (IDHM-Longevidade), educação (IDHMEducação) e renda (IDHM-Renda). O índice varia de zero (nenhum desenvolvimento humano) até 1 (desenvolvimento humano total), segundo o Programa das Nações Unidas Para o Desenvolvimento (PNUD), utilizado para classificação de países em desenvolvimento baixo ( 0 e 0,499$)$, médio $(0,500$ e 0,799$)$, alto $(0,800$ e 0,899$)$, muito alto $(0,900$ e 1$)$. O IDH de educação é avaliado a partir da média da taxa de alfabetização e da taxa bruta de frequência à escola, convertidas em índices, com peso 2 para o da taxa de alfabetização e peso 1 para o da taxa bruta de frequência ${ }^{19}$. A situação de higiene é um indicador utilizado pela SRH e avalia quanto em percentagem o município é ligado à rede geral de esgoto ${ }^{18}$.

\section{Análises estatísticas}

Foram realizadas análises descritivas dos dados, a partir da apuração de frequências simples absolutas e percentuais para as variáveis categóricas e organização dos resultados em mapas, tabelas e gráficos. Para comparação de diferenças e distribuição entre proporções, foram empregados teste $\mathrm{t}$ de Student e teste de Mann-Whitney. Foram realizadas associações entre as prevalências de esquistossomose e de enteroparasitos com diversos parâmetros socioeconômicos e educacionais utilizando-se cálculos de razão de prevalência e o intervalo de confiança a 95\% (IC 95\%). O nível de significância adotado em todas as análises foi de $5 \%(p<0,05)$. Essas análises foram feitas no software GraphPadPrisma.

\section{Considerações éticas}

O Projeto de Pesquisa foi aprovado pelo Comitê de Ética em Pesquisa da Universidade Federal de Sergipe, CAAE 0022.0.107.000-08.

\section{RESULTADOS}

Epidemiologia da esquistossomose no Estado de Sergipe no período de 2005 a 2008 segundo os dados do Programa de Controle da Esquistossomose

\section{Prevalência e distribuição geográfica}

Dos 75 municípios do Estado de Sergipe, havia dados do PCE relativos a 42 municípios em 2005, 46 em 2006, 48 em 2007 e 37 em 2008. Em todos os anos, $100 \%$ dos municípios analisados tiveram resultados positivos para infecção por $S$. mansoni.

O número de exames realizados em 2005 foi de 106.272, em 2006 foi de 145.259 , em 2007 foi de 134.502 e 78.854 em 2008. Essa 
redução no número de municípios e de exames realizados no ano de 2008 reflete o remanejamento dos agentes de saúde para outras atividades como o programa de controle da dengue, pois esta doença teve uma prevalência e morbidade elevada neste ano.

Na população estudada, foi frequente a presença de geo-helmintos sendo o Ascaris lumbricoides o mais comum (Figura 1).

A prevalência da esquistossomose no Estado de Sergipe, representada em termos de positividade parasitológica dos exames realizados pelo PCE foi de 13,6\% (14471/106287) de exames nos anos de 2005, 11,2\% (16196/145069) em 2006, 11,8\% (10220/86824) em 2007 e 10,6\% (8329/78859) em 2008.

Os mapas com as prevalências da esquistossomose, segundo os dados do PCE, nos anos de 2005 a 2008, nos municípios de Sergipe, são mostrados na Figura 2. A figura também mostra a delimitação das bacias hidrográficas do estado. As seis bacias hidrográficas do estado são as dos rios São Francisco, Vaza Barris, Real, Japaratuba, Sergipe e Piauí. Os rios São Francisco, Vaza-Barris e Real são rios federais porque atravessam mais de um estado. Enquanto os rios Japaratuba, Sergipe e Piauí são rios estaduais, pois suas bacias estão dentro do Estado de Sergipe sendo o Rio Japaratuba inteiramente sergipano ${ }^{18}$.

Em média, 57\% dos municípios enviaram dados para o PCE nesses 4 anos. Os municípios não avaliados por ausência de dados são mostrados nas áreas brancas dos mapas. Observa-se que os municípios afetados encontram-se distribuídos em todas as bacias hidrográficas do estado. No entanto, a bacia do São Francisco, que

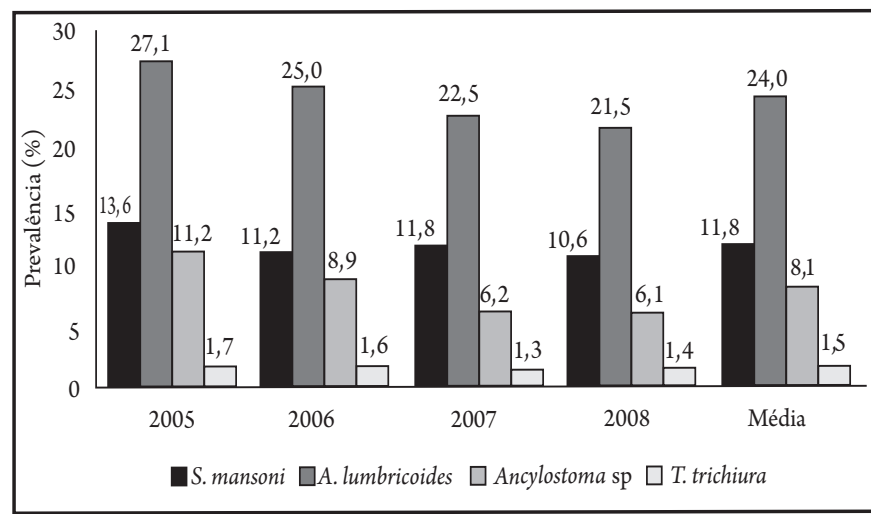

FIGURA 1 - Prevalência de enteroparasitos nos municípios do Estado de Sergipe de acordo com os dados do Programa de Controle da Esquistossomose (PCE) nos anos de 2005 a 2008.

corresponde a maior do estado, tem poucos municípios avaliados pelo programa, apesar de terem intensa atividade agrícola irrigada.

No ano de 2005, cinco municípios apresentaram prevalência $<5 \%, 34$ apresentaram prevalência entre 5 e $25 \%$ e 3 entre 25 e $50 \%$. Estes últimos foram os municípios de, Santa Luzia do Itanhi (29,6\%), São Cristovão (32,1\%) e Neópolis (35,3\%).

Em 2006, 8 municípios apresentavam prevalência $<5 \%, 34$ entre 5 e $25 \%$ e 4 entre 25 e $50 \%$. Estes últimos correspondem a Rosário do Catete (25,3\%), Indiaroba (25,6\%), São Cristovão $(34,2 \%)$ e Santa Rosa de Lima (41,3\%).

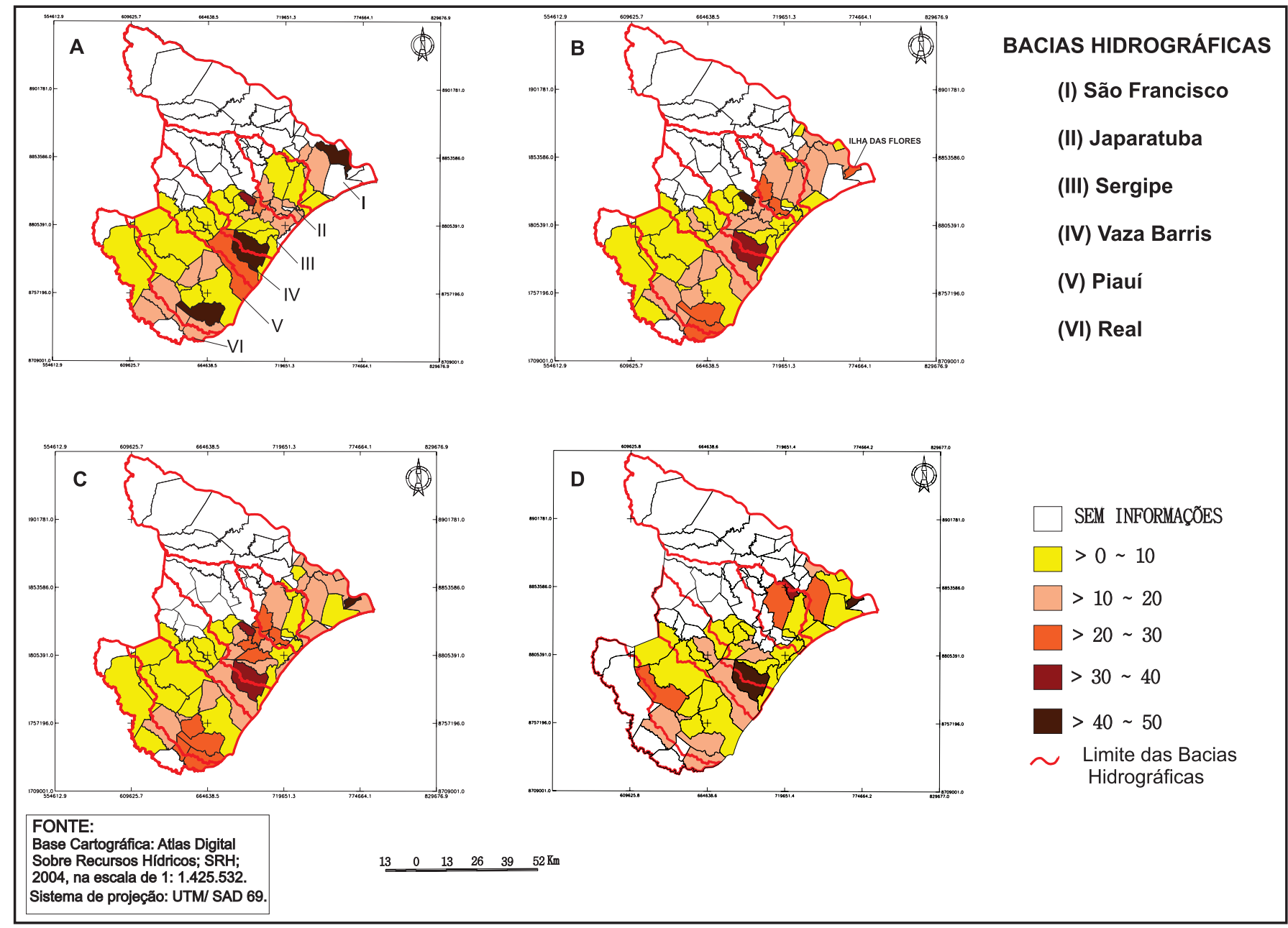

FIGURA 2 - Mapas representando as bacias hidrográficas e a distribuição da Prevalência da esquistossomose nos municípios do Estado de Sergipe nos anos de 2005 (A), 2006 (B), 2007 (C) e 2008 (D). 
Em 2007, 8 municípios apresentaram prevalência $<5 \%, 32$ entre 5 e $25 \%$ e 8 entre 25 e $50 \%$. Entre os últimos estavam Umbaúba $(25,5 \%)$, Riachuelo (28,9\%), Siriri $(29,6 \%)$, Japoatã $(30,3 \%)$, Maruim (30,7\%), Santa Rosa de Lima (32,1\%), São Cristovão (36,7\%) e Ilha das Flores (46,5\%).

Em 2008, 6 municípios apresentaram prevalência $<5 \%, 28$ entre 5 e $25 \%$ e 3 entre 25 e $50 \%$. Entre os últimos, estavam São Cristovão $(42,4 \%)$ Ilha das Flores (39,3\%) e Muribeca (29,2\%).

A Figura 3 mostra as prevalências dos municípios que em algum ano de avaliação apresentou prevalência acima de $20 \%$. Dos 75 municípios do estado, 37 a 48 (Média \pm SD $43.3 \pm 4$.8) enviaram dados para o PCE nos anos de 2005 a 2008. Uma média de 40\% ( $n=12)$ dos municípios que enviaram dados para o PCE apresentou prevalência acima deste valor. Sete dos 12 municípios apresentaram prevalências $\geq 30 \%$ e 3 tiveram prevalências $\geq 40 \%$ (Ilha das Flores, São Cristóvão e Santa Rosa de Lima). O número de municípios com altas positividades para S. mansoni aumentou progressivamente no decorrer dos anos de 2005 a 2007 em Sergipe. Entretanto, esta tendência parece não ser seguida em 2008.

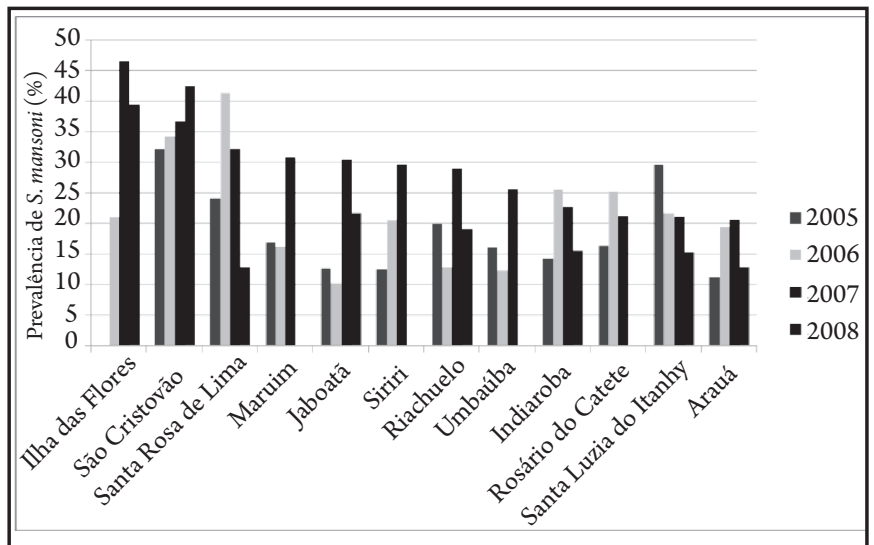

FIGURA 3 - Municípios do Estado de Sergipe com prevalência de Schistosoma mansoni acima de $\mathbf{2 0} \%$ de acordo com os dados do Programa de Controle da Esquistossomose (PCE) nos anos de 2005 a 2008.

Classificação da carga parasitária(CP) por ano nos municípios do Estado de Sergipe

Em 2005, 42 municípios do Estado de Sergipe notificaram casos de esquistossomose. A frequência de indivíduos com CP leve em Sergipe foi de 67,9\% (até 100 ovos/g de fezes), com CP moderada foi de $25,3 \%$ (101 a 400 ovos/g de fezes) e com CP elevada foi de 6,8\% (mais de 400 ovos/g de fezes). Os municípios de Itaporanga D’Ajuda e Santa Luzia do Itanhi apresentaram as maiores frequências de CP elevada, 3,9\% e 3,7\%, respectivamente. Em 2006, 46 dos 74 municípios do estado notificaram casos de esquistossomose. A frequência de indivíduos com CP leve em Sergipe foi de $68,4 \%$, com CP moderada foi de $24,2 \%$ e com CP elevada foi de $7,4 \%$. Os municípios de Santa Rosa de Lima e São Cristovão apresentaram as maiores frequências de CP elevada, 8,2\% e 5,4\%, respectivamente.

Em 2007, 48 dos 74 municípios do estado notificaram casos de esquistossomose. A frequência de indivíduos com CP leve em Sergipe foi de $64,7 \%$, com CP moderada $27,2 \%$ e com CP elevada $8,1 \%$. Os municípios de Ilha das Flores e São Cristovão apresentaram as maiores frequências de CP elevada, 10,9\% e 8\%, respectivamente.

Em 2008, 36 municípios notificaram casos de esquistossomose. A frequência de pessoas com CP leve em Sergipe foi de 9,3\%, $26 \%$ com CP moderada e $8 \%$ com CP elevada. Os municípios de Muribeca e
São Cristovão apresentaram as maiores frequências de CP elevada, $4,2 \%$ e $10,2 \%$, respectivamente.

\section{Porcentagem de tratados em Sergipe/ano}

A proporção de pessoas tratadas após o exame positivo para ovos de S. mansoni foi de 54,5\% (8.471/14469) em 2005, 74,4\% (12.106/16.267) em 2006, 73,5\% (10.897/14.828) em 2007 e 77,9\% (6.490/8.334) em 2008.

Fatores de risco socioeconômicos e ambientais associados às infecções por $S$. mansoni e geo-helmintos

Para estas análises, decidimos tomar como base os dados de 2007, considerando estes mais fidedignos por apresentarem maior número de municípios notificados. Neste ano, a prevalência de S. mansoni foi de $14,4 \%$ e a de outros parasitas foi de $22,3 \%$ para A. lumbricóides; 6,1\% para ancilostomídeos e 1,2\% de T. trichiura.

Foram comparados os municípios cujas prevalências para S. mansoni são maiores ou menores que $15 \%$ em relação a fatores socioeconômicos, ambientais e educacionais. Foi observada uma menor situação de higiene nos municípios com prevalências de S. mansoni acima de $15 \%$ (média $\pm \mathrm{DP}=6,6 \pm 11,50$ ) em relação aos municípios com prevalências menores que $15 \%(15,6 \pm 21,37)$, $\mathrm{p}=0,05$, teste de Mann-Whitney. Situação de higiene é um índice da Superintendência de Recursos Hídricos que mede a situação da rede de esgotos dos municípios. A média da situação de higiene dos municípios com maior prevalência de esquistossomose era de uma cobertura de $6,6 \%$ da rede de saneamento, enquanto que os municípios com menor prevalência possuíam uma média de 15,6\% de cobertura (Figura 4).

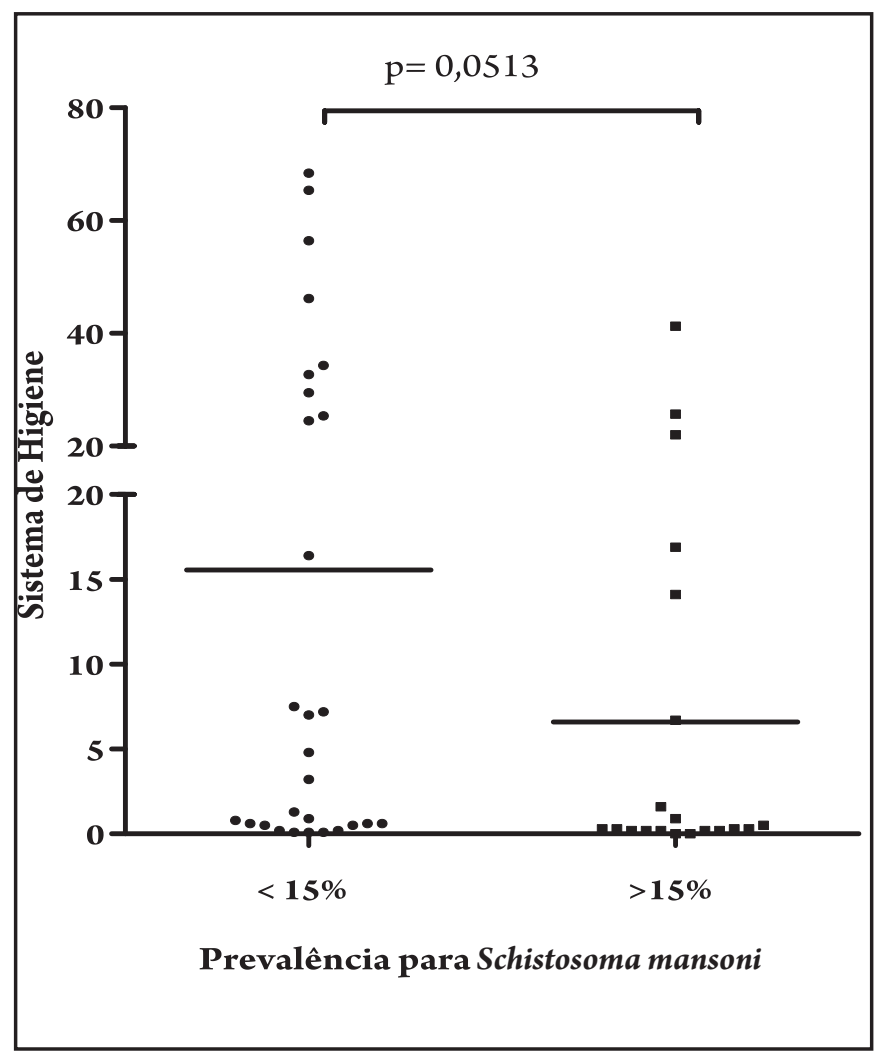

FIGURA 4 - Comparação entre as taxas de sistema de higiene avaliadas pela Superintendência de Recursos Hídricos do Estado de Sergipe nos municípios com prevalência de esquistossomose menor ou maior do que $15 \%$. $p$ teste de Mann-Whitney. 
Comparação entre os municípios sergipanos com prevalências de ancylostomideos $\geq 10 \%$ e $<10 \%$ em relação ao IDH de educação, demonstrou uma média menor nos municípios com prevalências $\geq 10 \%(0,71 \pm 0,01 ; \mathrm{n}=13)$ do que a dos municípios com prevalências $<10 \%(0,74 \pm 0,01 ; \mathrm{n}=35), \mathrm{p}=0,04$, teste t de Student. Os dados sugerem que o sistema de higiene influencia infecção por S. mansoni, enquanto a menor escolaridade influencia a infecção por ancilostomídeos (Figura 5).

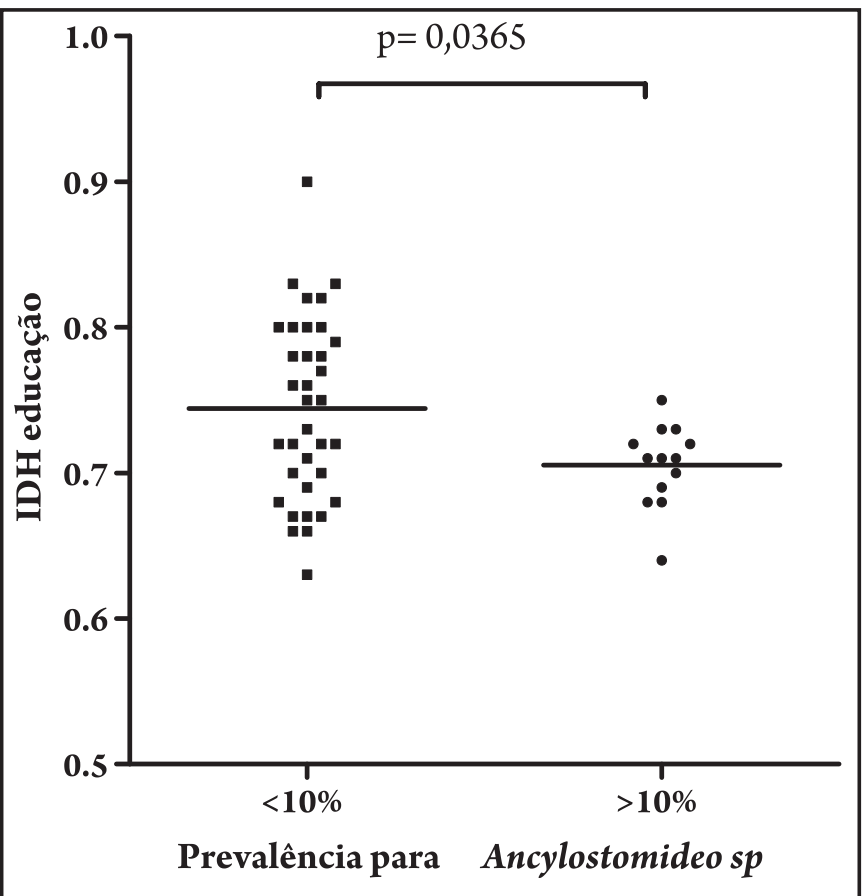

FIGURA 5 - Comparação entre os índices de desenvolvimento humano de educação avaliados pelo Instituto Brasileiro de Geografia e Estatística (IBGE), nos municípios com prevalência de ancilostomídeos menor ou maior do que 10\%. $p$ teste t de Student.

\section{DISCUSSÃO}

A esquistossomose, doença que acompanhou a humanidade desde quando esta habitava preferencialmente os campos, não foi, todavia, debelada com os progressos modernos. De fato, ela se encontra entre as poucas doenças parasitárias cuja distribuição mundial continua a aumentar. O próprio homem, ao modificar em seu benefício o espaço, fornece à doença um habitat novo, por exemplo, com a construção de represas e obras de irrigação ${ }^{20}$. Mesmo o processo de urbanização permite em seus centros periféricos, sem infraestrutura de saneamento básico e alimentados pelo processo migratório, a escalada da doença em um espaço inteiramente novo, dessa feita, urbano ${ }^{21}$.

Fatores ambientais influenciam de forma importante o estabelecimento da doença, devido às características do ciclo evolutivo do parasito que requer a eliminação de ovos das fezes no meio ambiente. No Brasil, ainda são comuns, especialmente nas zonas rurais pobres, condições favoráveis à infecção por $S$. mansoni, além de coinfecções entre S. mansoni e nematóides intestinais, tais como Ascaris lumbricoides, Trichuris trichiura e os ancilostomídeos.

Os resultados do estudo mostram que os dados do PCE apresentam uma cobertura insatisfatória dos municípios do Estado de Sergipe. O nordeste é importante reserva ecológica de hospedeiros intermediários de S. mansoni e o Estado de Sergipe é historicamente endêmico. Condições ambientais favoráveis e hospedeiros intermediários viabilizam a sobrevivência destes parasitos fora do hospedeiro humano, a exemplo da pobreza generalizada e os baixos níveis de higiene e de saneamento básico ${ }^{20}$. A detecção de alta prevalência de geo-helmintos em muitos municípios estudados sugere que o programa de controle da esquistossomose pode ser também utilizado para diagnóstico e controle não apenas da esquistossomose, como também de geohelmintos como A. lumbricóides e ancilostomídeos.

O IDH de todos os municípios de Sergipe é compatível com municípios em desenvolvimento. Entretanto, a esquistossomose mansônica está presente mesmo em Cidades como Aracaju e São Cristóvão que possuem IDH semelhantes aos de países desenvolvidos. Como a doença é habitualmente focal, estes dados sugerem que alguns seguimentos dessas populações estão sujeitas a condições sanitárias que afrontam a dignidade humana e possibilitam a persistência da doença $a^{21,22}$.

A situação de higiene, índice que reflete o percentual de esgotamento sanitário, tem influência no grau de contaminação ambiental por ovos de S. mansoni dos municípios, ou seja, onde há maior rede de esgotos, a prevalência da esquistossomose é menor. Assim como os municípios com taxas de infecção por ancilostomídeos menores de $10 \%$ são aqueles onde existe um maior IDH educacional, sugerindo a influência da educação no controle desta doença, com melhora dos hábitos de higiene pessoal e de uso de calçados.

A subnotificação da doença e a coleta de dados não representativos da população podem levar a dados incorretos de prevalência tanto para valores mais baixos como mais elevados. Segundo Katz e Peixoto ${ }^{2}$, a ausência de dados precisos indica a necessidade de adequado levantamento nacional da prevalência da esquistossomose. Para a estimativa segura da prevalência da esquistossomose, necessita-se de levantamentos parasitológicos feitos com amostragem adequada, em nível nacional, levando em consideração que a prevalência da parasitose não apresenta distribuição regular e sim focal ${ }^{2}$. A avaliação parasitológica do PCE é realizada por agentes de saúde dos municípios afetados, sem uma espacialização adequada, justificando dados de prevalência significantemente elevadas em municípios como São Cristóvão e Aracaju, onde provavelmente a coleta de amostras foi realizada nos locais próximos às fontes de contato, superestimando a prevalência geral. Este aspecto justifica a ausência de associação entre IDH com a infecção. A execução do PCE é de responsabilidade municipal; entretanto, municípios com índice de positividade branda podem se valer do recurso de implantação de um sistema de vigilância que é indicada para municípios em que a positividade é inferior a $25 \%$ sendo necessário, entretanto, que metade das localidades do município tenha positividade inferior a $5 \%{ }^{23}$. Nestes casos, a avaliação será feita a partir dos casos detectados na rede básica de saúde e não necessariamente através de busca ativa como propõe o PCE.

Dados da rede básica de saúde coletados sem um inquérito epidemiológico sistemático também podem não corresponder à realidade do município, além de não serem enviados para o PCE, apesar de serem registrados no Sistema Nacional de Agravos de Notificação (SINAN).

O presente estudo mostra o mapeamento das prevalências de esquistossomose no Estado de Sergipe, sendo todos os municípios avaliados pelo PCE afetados pela doença. Doze destes municípios apresentam prevalências acima de $20 \%$ em algum ano de avaliação, chegando à prevalência de 45,5\% (Ilha das Flores). Algumas destas áreas já foram consideradas áreas endêmicas em período pregresso, como os municípios das bacias dos rios Piauí e Real. A despeito 
disto, a prevalência e morbimortalidade reduziu em relação ao passado, antes da implantação do Programa especial de Controle da Esquistossomose (PECE), devido aos tratamentos de massa instituídos. O PECE foi iniciado em Sergipe em 1976, quando se verificou uma prevalência em escolares de 7 a 14 anos que variou entre $10,9 \%$, a $78,5 \%$, com média de $40,6 \%$ em diversos municípios, com maiores prevalências nas regiões banhadas pelos rios Sergipe, Japaratuba e Vaza-Barris ${ }^{13}$.

Atualmente, os dados do PCE levantados neste trabalho mostram que a prevalência geral do estado vem se mantendo estabilizada, sendo $13,6 \%, 11,2 \%, 11 \%$ e $10,6 \%$ em 2005, 2006, 2007 e 2008 , respectivamente. No entanto, as cargas parasitárias documentadas são ainda elevadas, sendo as frequências de cargas parasitárias moderadas e altas ( $>100$ ovos/g de fezes) acima de $30 \%$ em todos os anos analisados. Considerando os dados de Sleigh cols que mostram morbidade da doença em indivíduos com cargas parasitárias acima de 100 ovos/g, há riscos de desenvolvimento de formas graves em nossa população ${ }^{10}$.

Os dados do estudo atual mostram uma alta prevalência de infecção por S. mansoni no Estado de Sergipe, com alta carga parasitária, demonstrando a necessidade de intensificar a execução das medidas de controle, especialmente aquelas voltadas ao saneamento básico. Os municípios de maior risco estão nas bacias hidrográficas mais importantes do estado, as do São Francisco e Vaza-Barris, o que pode constituir subsídio para o direcionamento de medidas de controle nessas áreas.

Outro aspecto importante é o crescente número de casos notificados em cidades de maior porte como São Cristóvão e Aracaju, o que também vem ocorrendo em outros estados. Em Belo Horizonte (MG) e Salvador (BA), por exemplo, há focos naturais de infecção pelo $S$. mansoni, possivelmente como resultado da migração de indivíduos de regiões endêmicas para estas cidades, além das deficiências de instalação de infraestrutura de saneamento básico nas áreas periurbanas mais pobres dessas cidades ${ }^{21,22,24,25}$.

O controle da transmissão vai além da capacidade dos profissionais de saúde e do tratamento em massa e deve ser feito com ações governamentais que combinem instalação de água e esgoto nas casas, mudanças no meio ambiente, educação sanitária, combate aos caramujos, além do diagnóstico e tratamento das pessoas infectadas ${ }^{2}$.

Sendo a esquistossomose uma doença focal, as técnicas de espacialização utilizadas em nosso estudo merecem ser incorporadas com a atual metodologia utilizada pela Secretaria de Vigilância Sanitária para otimizar a determinação de áreas de risco e repasse financeiro para controle da esquistossomose.

\section{CONFLITO DE INTERESSE}

Os autores declaram não haver nenhum tipo de conflito de interesse no desenvolvimento do estudo.

\section{SUPORTE FINANCEIRO}

MS/CNPq/FAPITEC/SE/SES N06/2007, PIBIC/CNPq. CVVR é bolsista da CAPES; ARJ e RPA são pesquisadores do CNPq.

\section{REFERÊNCIAS}

1. Chitsulo L, Engels D, Montresor A, Savioli L. The global status of schistosomiasis and its control. Acta Trop 2000; 77: 41-51.

2. Katz N, Peixoto SV. Critical analysis of the estimated number of Schistosomiasis mansoni carriers in Brazil. Rev Soc Bras Med Trop 2000; 33:303-308.
3. Amaral RS, Tauil PL, Lima DD, Engels D. An analysis of the impact of the Schistosomiasis Control Programme in Brazil. Mem Inst Oswaldo Cruz 2006; 101(suppl 1):79-85.

4. Cheever AW. Pathogenesis of Schistosoma mansoni infection. Mem Inst Oswaldo Cruz 1992; 87 (suppl 4):337-340.

5. Coura JR, Amaral RS. Epidemiological and control aspects of schistosomiasis in Brazilian endemic areas. Mem Inst Oswaldo Cruz 2004; 99:13-19.

6. Coura JR, Conceicao J, dos Santos ML, de Mendonca ZG, Cutrim RN. Cross-sectional and evolutive studies of schistosomiasis mansoni in untreated and mass treated endemic areas in the southeast and northeast of Brazil. Mem Inst Oswaldo Cruz 1992; 87 (suppl 4):175-182.

7. Jesus AM, Almeida RP, Bacellar O, Araujo MI, Demeure C, Bina JC, et al Correlation between cell-mediated immunity and degree of infection in subjects living in an endemic area of schistosomiasis. Eur J Immunol 1993; 23:152-158.

8. Jesus AR, Magalhaes A, Miranda DG, Miranda RG, Araujo MI, de Jesus AA, et al. Association of type 2 cytokines with hepatic fibrosis in human Schistosoma mansoni infection. Infect Immun 2004; 72:3391-3397.

9. Lambertucci J, Silva L, Voieta I. Esquistossomose Mansônica. In: Coura JR, editor Dinâmica das Doenças Infecciosas e Parasitárias. Rio de Janeiro: Guanabara Koogan; 2005. p. 931-946.

10. Sleigh AC, Hoff R, Mota EA, Sherlock I, Mott KE, Barreto ML, et al. Three-year propective study of evolution of Manson's Schistosomiasis in Brazil. Lancet $1985 ; 2: 63-66$.

11. Butterworth AE, Fulford AJ, Dunne DW, Ouma JH, Sturrock RF. Longitudinal studies on human schistosomiasis. Philos Trans R Soc Lond B Biol Sci $1988 ; 321: 495-511$

12. Rihet P, Demeure CE, Bourgois A, Prata A, Dessein AJ. Evidence for an association between human resistance to Schistosoma mansoni and high anti-larval IgE levels. Eur J Immunol 1991; 21:2679-2686.

13. Coura-Filho P. Schistosomiasis mansoni occurrence in urban territory. 1. A case study of a peripheral urban area in Belo Horizonte, Minas Gerais, Brazil. Cad Saude Publica 1997; 13: 245-255.

14. Campos MR, Valencia LI, Fortes Bde P, Braga RC, Medronho Rde A. Spatial distribution of Ascaris lumbricoides infection. Rev Saude Publica 2002; 36:69-74.

15. Instituto Brasileiro de Geografia e Estatística [Internet]. IBGE Cidades. 2010 [acesso 09 jan 2010]. Disponível em: http://www.ibge.gov.br/cidadesat/ topwindow.htm?1/

16. Waldman EA, Silva LJ, Monteiro CA. Trajetórias das doenças infecciosas: da eliminação da poliomielite à introdução da cólera. In: Monteiro CA, editor. Velhos e novos males da saúde no Brasil: a evolução do país e de suas doenças. $2^{a}$ ed. São Paulo: Editora Hucitec. Universidade de São Paulo; 2000. p. 195-244.

17. World Health Organization. The Control of Schistosomiasis. Report of a WHO Expert Committee. Geneva: World Health Organization; 1985.

18. Superintendência de Recursos Hídricos [Internet]. Superintendência de Recursos Hídricos. 2010 - [acesso 11 abril 2010]. Disponível em: http://www.seplantecsrh.se.gov.br/.

19. Programa das Nações Unidas Para o Desenvolvimento [Internet]. Programa das Nações Unidas para o Desenvolvimento. Atlas de Desenvolvimento Humano. 2000 - [acesso 11 abril 2010]. Disponível em: http://www.pnud.org.br/idh/.

20. Coutinho A, Silva M, Gonçalves J. Estudo Epidemiológico de Esquistossomose Mansônica em Áreas de Irrigação do Nordeste Brasileiro. Cad Saude Publica 1992; 8:302-310

21. Guimarães I, Tavares-Neto J. Transmissão urbana de esquistossomose em crianças de um bairro de Salvador, Bahia. Rev Soc Bras Med Trop 2006; 39:451-455.

22. Rollemberg C, Quintans J, Santos R. Avaliação do Programa de Controle de Esquistossomose no Bairro Santa Maria, Aracaju, Sergipe, sob a perspectiva farmacêutica. Rev FAPESE 2008; 4:63-82.

23. Ministério da Saúde. Controle da Esquistossomose Brasília. Diretrizes Técnicas Brasília: Fundo Nacional Saúde.Ministério da Saúde; 1998.

24. Drummond S, Silva L, Amaral R, Souza-Pereira S, Antunes C, Lambertuci J. Morbidity of schistosomiasis mansoni in the state of Minas Gerais, Brazil. Mem Inst Oswaldo Cruz 2006; 101:37-44.

25. Geiger S. Immuno-epidemiology of Schistosoma mansoni infections in endemic populations co-infected with soil-transmitted helminths: Present knowledge, challenges, and the need for further studies. Acta Trop.2008; 108:118-123. 